\title{
Numerical Solutions of the Hattendorff Differential Equation for Multi-state Markov Insurance Models
}

\author{
Nathan Ritchey ${ }^{\mathrm{a}}$ and Rajeev Rajaram ${ }^{\mathrm{b}}$ \\ ${ }^{a, b}$ Kent State University, Dept. of Math. Sci., Kent, OH - 44145, USA;
}

\begin{abstract}
ARTICLE HISTORY
Compiled December 8, 2021

ABSTRACT

We provide methodology and numerical results for the Hattendorff differential equation for the continuous time evolution of the variance of $L_{t}^{(j)}$, the loss at time $t$ random variable for a multi-state process, given that the state at time $t$ is $j$.
\end{abstract}

\section{KEYWORDS}

Policy Value; Kolmogorov forward equations; Multi-state model; Thiele's differential equation; Hattendorff differential equation

\section{Introduction}

The original Hattendorff theorem in Hattendorff (1868) states that in a life insurance policy, the losses in different years are uncorrelated and have zero means, hence the variance of the total loss is the sum of variances of the losses in individual years. This result has had a revival in modern day actuarial mathematics with a flavor of stochastic processes or a martingale approach as seen in Gerber $(1979,1986)$, Papatriandafylou and Waters (1984), Ramlau-Hansen (1988), Norberg (1992). Furthermore, the theorem has been mentioned in classic textbooks such as Bowers et al. (1986). More recently in Bladt et al. (2020) a matrix representation was carried out to obtain representation formulas for all higher order moments for the loss at time $t$ of the random variable $L_{t}$.

In a previous paper, Rajaram and Ritchey (2021), we presented an explicit derivation of a differential equation (which we named as the Hattendorff Differential Equation) that describes the evolution of the variance of $L_{t}$ in the continuous time case. Along the way, we derived an explicit recursion formula for the discrete time case for annual and $h$-yearly cash flows. In this paper, we exploit the derived representation of the continuous time differential equation using MATLAB and compute ${ }^{2} \sigma_{t}^{(j)}$ as the solution of a backwards in time differential equation. We demonstrate this process by solving examples of several instances of a multi-state model in which the practicioner can use as an example for their own specific multi-state model.

The main goals of this paper are as follows:

(1) Employ the differential equation that was derived in the the previous paper that governs the continuous time evolution of the variance of the loss at time

Nathan Ritchey Email: nritche2@kent.edu

Rajeev Rajaram Email: rrajaram@kent.edu 
$t$ random variable given that the state at time $t$ is $j$ (denoted by ${ }^{2} \sigma_{t}^{(j)}$ ), for a general setting of benefits and premiums in the setting of a multistate Markov insurance model, to numerically solve specific examples.

(2) Demonstrate how Matlab can be used to solve examples when they arise for the end user.

(3) Provide tips and advice on solving the Hattendorf differential equation.

As promised in the previous paper, Rajaram and Ritchey (2021), this paper provides the actual computation/approximation of ${ }^{2} \sigma_{t}^{(j)}$ for all time $t$ as a matter of solving a system of coupled differential equations. To our knowledge, this is the first time numerical solutions of this general insurance model for computation of the variance of loss ${ }^{2} \sigma_{t}^{(j)}$ have been considered.

In line with Dickson et al. (2020)-Chapter 8, we consider a multi-state model with $m+1$ states labeled as $0,1, \ldots, m$ with instantaneous transitions possible between pairs of states. We use $Y(t)$ to represent the state at time instant $t .\{Y(t)\}_{t \geq 0}$ is a stochastic process for $t \geq 0$ (continuous or discrete time depending on when we choose to make observations), with $Y(t)=i$ to mean that the individual is in state $i$ at age $x+t$ (initial age is taken as $x$ ).

We introduce the following notation for states $i$ and $j$ and ages $x \geq 0$ as in Dickson et al. (2020)-Chapter 8:

\section{Notation 1.}

(1) ${ }_{t} p_{x}^{i j}=\operatorname{Pr}[Y(x+t)=j \mid Y(x)=i]$

(2) $t p_{x}^{\overline{i i}}=\operatorname{Pr}[Y(x+s)=i$ for all $s \in[0, t] \mid Y(x=i)]$

(3) Force of transition: $\mu_{x}^{i j}=\lim _{h \rightarrow 0^{+}} \frac{h p_{x}^{i j}}{h}$ for $i \neq j$

(4) $L_{t}^{(j)}=$ Future loss random variable for a policy which is in state $j$ at time $t$

(5) $V_{t}^{(j)}=E\left[L_{t}^{(j)}\right]$

(6) ${ }^{2} \sigma_{t}^{(j)}=\operatorname{Var}\left[L_{t}^{(j)}\right]$

For simplicity of notation we will simply write $Y(t)$ instead of $Y(x+t)$, with the assumption that the policyholder is of age $x$ at $t=0$. We also make the following assumptions as in Dickson et al. (2020)-Chapter 8:

\section{Assumption 1.}

(1) (Markovian assumption) For any states $i, j$ and times $t$ and $t+s$, the conditional probability $\operatorname{Pr}[Y(t+s)=j \mid Y(t)=i]$ is well defined and is independent of the knowledge of the process before time $t$.

(2) The probability of two or more transitions in a time interval $h$ is $o(h)$.

(3) For all states $i$ and $j$ and all ages $x \geq 0$, we assume that ${ }_{t} p_{x}^{i j}$ is a differentiable function of $t$.

(4) For simplicity, we assume that there are no expenses although expenses can be easily incorporated into the formulation.

(5) For simplicity, we assume a constant interest rate $i$ although a variable interest rate can be easily incorporated into the formulation.

We also make the following assumptions and notation on the payments for $h$-yearly cash flows:

\section{Notation 2.}


(1) We assume that for the discrete time payments, the payments depend at most on the state of the process $Y(t)$ at the start and end of the period between cash flows.

(2) $h P_{t}^{(j)}$ - amount of premium payable at the start of the interval $(t, t+h)$ given that $Y(t)=j . P_{t}^{(j)}$ is a premium rate which is differentiable with respect to $t$.

(3) $h B_{t+h}^{(k)}$ - benefit payable at the end of the interval $(t, t+h)$ given that $Y(t+h)=k$. $B_{t}^{(k)}$ is a benefit rate which is differentiable with respect to $t$. Also, for consistency we have

$$
\lim _{h \rightarrow 0} B_{t+h}^{(k)}=-P_{t}^{(k)}
$$

We allow for the mathematical possibility that $B_{t+h}^{(k)}$ and $P_{t}^{(k)}$ both exist in the time interval $(t, t+h)$ as benefit rate at the end given $Y(t+h)=k$ and premium rate at the beginning given $Y(t)=k$ respectively, with the understanding that one or both can be zero for a given state $k$.

(4) $S_{t+h}^{(j k)}$ - lump sum benefit payable at the end of the interval $(t, t+h)$ if $Y(t+j)=k$, given that $Y(t)=j$. Here we allow $S_{t+h}^{(j k)}$ to be differentiable with respect to $t$ and also allow $j=k$ as a mathematical possibility as well, with the assumption that for small $h, S_{t+h}^{(j j)}=h B_{t+h}^{(j)}$, and hence

$$
\lim _{h \rightarrow 0} S_{t+h}^{(j j)}=0 ; \quad \lim _{h \rightarrow 0} \frac{S_{t+h}^{(j j)}}{h}=B_{t}^{(j)} .
$$

We include terms of the form $S_{t}^{(j j)}$ for symmetry in the derivation, with the understanding that $S_{t}^{(j j)}=0$ since $S_{t}^{(j k)}$ is in principle, a benefit paid upon transition from state $j$ to state $k$ with $j \neq k$, and hence is equal to zero when $j=k$.

(5) We define $W_{t+h}^{(j k)}=V_{t+h}^{(k)}+h B_{t+h}^{(k)}-S_{t+h}^{(j k)}$ and $W_{t}^{(j k)}=\lim _{h \rightarrow 0} W_{t+h}^{(j k)}=V_{t}^{(k)}-S_{t}^{(j k)}$.

We also introduce notation that will be useful in Section 2 - Section 4 .

\section{Notation 3.}

(1) Represent the variance vector as

$$
\overrightarrow{{ }^{2} \sigma_{t}}=\operatorname{Var}\left(L_{t}\right)=\left[{ }^{2} \sigma_{t}^{(0)},{ }^{2} \sigma_{t}^{(1)}, \ldots,{ }^{2} \sigma_{t}^{(m)}\right]^{T}
$$

(2) Let the $(i, j)$ th element of the matrix ${ }_{h} \mathbb{P}$ be given by

$$
\left({ }_{h} \mathbb{P}_{x+t}\right)_{i j}={ }_{h} p_{x+t}^{i j} .
$$

(3) In this light, we have

$$
{ }^{2} \sigma_{t+h}^{(j), d}=\operatorname{Var}\left(L_{t+h}^{(j), d}\right)=v^{2 h} \sum_{k \neq l}{ }_{h} p_{x+t h}^{j k} p_{x+t}^{j l}\left(W_{t+h}^{(j k)}-W_{t+h}^{(j l)}\right)^{2},
$$


where

$$
L_{t+h}^{(j), d}=v^{h} W_{t+h}^{(j k)} \quad \text { with probability }{ }_{h} p_{x+t}^{j k} \quad \text { and } \quad \sum_{k=0}^{m}{ }_{h} p_{x+t}^{j k}=1 .
$$

(4) Define the vector of variance of losses as

$$
\overrightarrow{{ }^{2} \sigma_{t}^{d}}=\operatorname{Var}\left(L_{t}^{d}\right)=\left[{ }^{2} \sigma_{t}^{(0), d},{ }^{2} \sigma_{t}^{(1), d}, \ldots,{ }^{2} \sigma_{t}^{(m), d}\right]^{T}
$$

(5) Define the $j$-th element of the vector $\overrightarrow{\mu_{x+t}^{d}}$ as

$$
\left\{\overrightarrow{\mu_{x+t}^{d}}\right\}_{j}=\sum_{l \neq j} \mu_{x+t}^{j l}\left(W_{t}^{(j j)}-W_{t}^{(j l)}\right)^{2} .
$$

The paper is organized as follows. In Section 2, we state some of the important theorems from the previous paper for discrete recursive relationships for ${ }^{2} \sigma_{t}^{(j)}$. We present terminal conditions and an introduction to numerical computations in Section 3. In Section 4, we present and solve three particular models that can serve as a roadmap on how Matlab can be used to solve the differential equation describing the evolution of ${ }^{2} \sigma_{t}^{(j)}$ by using the $h$-yearly recursion stated in Section 2 . We then present some details in Section 5 about how Matlab is used to solve the problems in Section 4. Finally, we conclude with some remarks in Section 6.

\section{Theorems for ${ }^{2} \sigma_{t}^{(j)}$}

In this section, we list two important theorems from our previous paper, Rajaram and Ritchey (2021), which provide an explicit annual relationship for ${ }^{2} \sigma_{t}^{(j)}$ that mirrors the traditional Hattendorff recursion for the alive-dead model, but includes the option for multiple states.

Theorem 2.1. Assume that Notation 1, Assumption 1 and Notation 2 are true. Then the following explicit annual relationship holds for ${ }^{2} \sigma_{t}^{(j)}$ :

$$
\begin{aligned}
{ }^{2} \sigma_{t+m}^{(j)}=v^{2 s} \prod_{i=0}^{s-1} p_{x+t+i}^{j j} \cdot{ }^{2} \sigma_{t+s}^{(j)}+\sum_{n=1}^{s} v^{2 n}\left(\prod_{i=0}^{n-2} p_{x+t+i}^{j j}\right) \sum_{\substack{k=0 \\
k \neq j}}^{i} p_{x+t+n-1}^{j k} \cdot{ }^{2} \sigma_{t+n}^{(k)} \\
+\sum_{n=1}^{s} v^{2 n}\left(\prod_{i=0}^{n-2} p_{x+t+i}^{j j}\right) \sum_{k \neq l}^{i} p_{x+t+n-1}^{j k} \cdot p_{x+t+n-1}^{j l}\left[\left(W_{t+n}^{(j k)}-W_{t+n}^{(j l)}\right]^{2} .\right.
\end{aligned}
$$

Here is the continuous time differential equation satisfied by ${ }^{2} \sigma_{t}^{(j)}$. 
Theorem 2.2. Assume that Notation 1, Assumption 1 and Notation 2 are true. Then ${ }^{2} \sigma_{t}^{(j)}$ satisfies the following differential equation:

$$
\begin{array}{r}
\frac{d}{d t}{ }^{2} \sigma_{t}^{(j)}=\left(2 \delta+\sum_{k \neq j} \mu_{x+t}^{j k}\right) \cdot{ }^{2} \sigma_{t}^{(j)}-\sum_{k \neq j} \mu_{x+t}^{j k} \cdot{ }^{2} \sigma_{t}^{(k)} \\
-\sum_{l \neq j} \mu_{x+t}^{j l}\left(W_{t}^{(j j)}-W_{t}^{(j l)}\right)^{2} .
\end{array}
$$

Note that we can use matrices to represent the theorems that were presented above.

\section{Terminal conditions}

In this section, we introduce terminal conditions and outline the steps involved to compute ${ }^{2} \sigma_{t}$ numerically.

\subsection{N year term or endowment insurance}

We consider end conditions for an $N$ year term (no survival benefit) or endowment insurance (survival benefit). In this case, all benefits at the end of the term are certain and hence the variance equals 0 . That is, ${ }^{2} \sigma_{N}=\overrightarrow{0}$.

\subsection{Numerical Solutions}

We use Matlab to solve specific instances of this model. Of course, any of the wellknown solvers can be used. It is important to note that solving instances of this problem requires solving either two systems of differential equations, first solving one then inputting results into the other, or solving one larger coupled system. Because of the size of most applicable problems, it is quite efficient to simply solve the larger system of differential equations. Also, this system of differential equations has terminal conditions, as opposed to initial conditions. Most commercial codes require inital conditions. For our model, we have a backwards in time system. Thus, we can either develop a program that manages this, or use a change of variable technique to transform the terminal conditions to initial conditions. Since the change of variable is quite straight forward, we chose this route so that the most basic solver could be used.

To transform our problem, let $s=t_{0}-t$ where $t_{0}$ is the length of a policy. We also need to use the Chain Rule for this transformation and note that $\mathrm{dt} / \mathrm{ds}=-1$.

\section{Computational Examples}

In this section, we present four examples, each with an increasing number of states. We use Matlab to numerically approximate the solutions to these problems. 


\subsection{Example 1: Continuous time classic insurance model}

For the first model, a two state model (alive and dead), we use an example that was found as Example 7.13, Bowers et al. (1986). In this example, a twenty-year endowment insurance issued to a life aged 30 . The insured value is $\$ 100,000$ and payable immediately on death, or on survival to the end of the term, whichever occurs sooner. Premiums are payable continuously at a constant rate of $\$ 2500$ per year throughout the term of the policy. The policy value basis uses a constant force of interest, $\delta=0.04$ per year, and makes no allowance for expenses. In this example, we calculate the value $V_{10}$ and its standard deviation $\sqrt{{ }^{2} \sigma_{10}}$ using the general model that was presented in Rajaram and Ritchey (2021).

Using this information $n=20, P=2500$, age $=30$, and $S=100,000$ we calculate $\mu_{50}=0.0015$. Thus, the problem is to solve the system of differential equations:

$$
\begin{aligned}
V_{t}^{\prime} & =.04115 V_{t}+2385 \\
\sigma_{t}^{\prime} & =.08115 \sigma_{t}-.00115\left(V_{t}-100000\right)^{2}
\end{aligned}
$$

Using Matlab, the solution is shown in Figure 1.

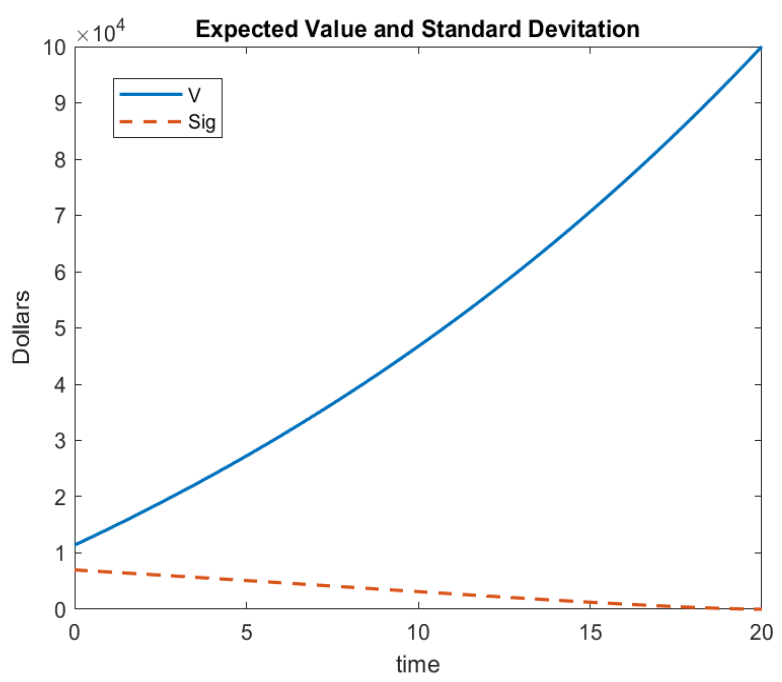

Figure 1

Also,

$$
V_{10} \approx 46,692 \text { and } \sqrt{{ }^{2} \sigma_{10}} \approx 3461
$$

Note that $V_{10}$ from this model agrees with that which is calculated in the textbook. 


\subsection{Example 2: Continuous time disability income insurance model}

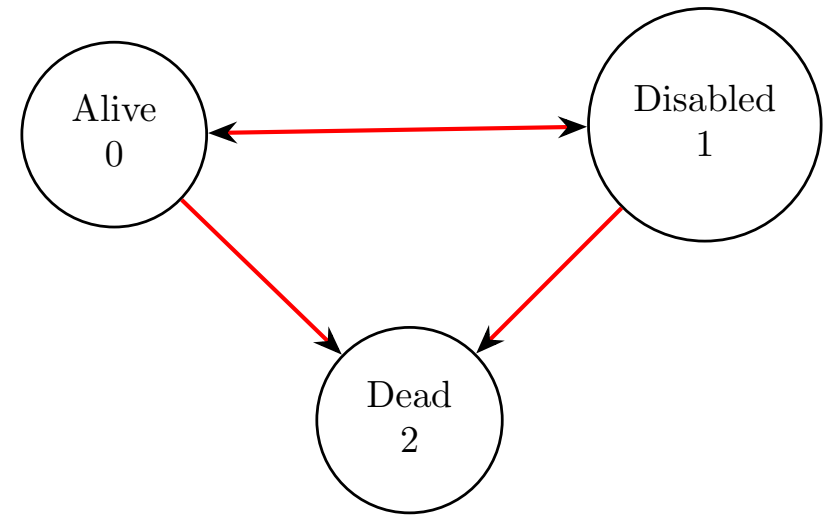

Figure 2

Consider a disability income insurance model with three states, alive, temporary disability, and dead, as shown in Figure 2. We also assume that the following transition rates apply to this example:

$$
\mu_{60+t}^{01}=0.05, \quad \mu_{60+t}^{02}=0.25 t, \quad \mu_{60+t}^{10}=0.25, \quad \mu_{60+t}^{12}=0.04 t .
$$

The valuation force of interest is $5 \%$. You are also given:

$$
\bar{a}_{60}^{00}=5.1716, \quad \bar{a}_{60}^{01}=0.8430, \quad \bar{A}_{60}^{02}=0.6980, \quad \bar{a}_{60}^{11}=4.8201, \quad \bar{A}_{60}^{12}=0.7350,
$$

$\bar{a}_{70}^{00}=2.4769, \quad \bar{a}_{70}^{01}=0.2012, \quad \bar{A}_{70}^{02}=0.8659, \quad \bar{a}_{70}^{10}=0.1051, \quad \bar{a}_{70}^{11}=1.8528, \quad \bar{A}_{70}^{12}=0.9017$,

and

$$
{ }_{10} p_{60}^{00}=0.18314,{ }_{10} p_{60}^{01}=0.06181
$$

A 10-year health insurance product has the following features:

- The product is issued to lives aged 60 in the healthy state.

- The product pays a death benefit of $\$ 5000$ at the moment of death.

- The product pays a continuous disability benefit at a rate of $\$ 750$ per year while the insured is temporarily disabled.

- Net premiums are payable continuously while the insured is healthy.

- The product pays an endowment of $\$ 1000$ if the person lives beyond 10 years.

The net premium can be computed using the Equivalence Premium Principle that is assumed to be at a rate of $P=\$ 695.64$.

By Thiele's differential equation, we have the following: 


$$
\begin{aligned}
\frac{d}{d t}\left(V_{t}^{(0)}\right) & =\delta V_{t}^{(0)}+P-\left\{\mu_{60+t}^{01}\left(S_{t}^{(01)}+V_{t}^{(1)}-V_{t}^{(0)}\right)+\mu_{60+t}^{02}\left(S_{t}^{(02)}+V_{t}^{(2)}-V_{t}^{(0)}\right)\right\} \\
& =(0.05) \cdot V_{t}^{(0)}+695.64-\left\{0.05\left(0+V_{t}^{(1)}-V_{t}^{(0)}\right)+0.025 t\left(5000+V_{t}^{(2)}-V_{t}^{(0)}\right)\right\} \\
& =695.64+V_{t}^{(0)}(0.05+0.05+0.025 t)-0.05 V_{t}^{(1)}-(0.025 t)(5000) \\
& =695.64+V_{t}^{(0)}(0.1+0.025 t)-0.05 \cdot V_{t}^{(1)}-125 t .
\end{aligned}
$$

Note that $V_{t}^{(2)}=0$ for all $t \geq 0$.

We also have the following for $V_{t}^{(1)}$ :

$$
\begin{aligned}
\frac{d}{d t}\left(V_{t}^{(1)}\right) & =\delta V_{t}^{(1)}-B_{t}^{(1)}-\left\{\mu_{60+t}^{10}\left(S_{t}^{(10)}+V_{t}^{(0)}-V_{t}^{(1)}\right)+\mu_{60+t}^{12}\left(S_{t}^{(12)}+V_{t}^{(2)}-V_{t}^{(1)}\right)\right\} \\
& =(0.05) \cdot V_{t}^{(1)}+750-\left\{0.025\left(0+V_{t}^{(0)}-V_{t}^{(1)}\right)+0.04 t\left(5000-V_{t}^{(1)}\right)\right\} \\
& =(0.05+0.025+0.04 t) \cdot V_{t}^{(1)}-0.025 V_{t}^{(0)}-750-200 t \\
& =(0.075+0.04 t) \cdot V_{t}^{(1)}-0.025 V_{t}^{(0)}-(750-200 t) .
\end{aligned}
$$

Since $V_{t}^{(2)}=0$ for all $t \geq 0$ we have no need for a differential equation.

Thus, Thiele's differential equation can be written as

$$
\begin{aligned}
\frac{d}{d t}\left[\begin{array}{l}
V_{t}^{(0)} \\
V_{t}^{(1)}
\end{array}\right]= & {\left[\begin{array}{cc}
0.1+0.025 t & -0.05 \\
-0.025 & 0.075+0.04 t
\end{array}\right]\left[\begin{array}{c}
V_{t}^{(0)} \\
V_{t}^{(1)}
\end{array}\right]+\left[\begin{array}{c}
-125 t \\
695.64-(750+200 t)
\end{array}\right], } \\
& \text { Terminal Conditions: }\left\{\begin{array}{l}
V_{10}^{(0)}=1000 \\
V_{10}^{(1)}=0
\end{array} \quad\right. \text { (Endowment) }
\end{aligned}
$$

Now let's consider Hattendorff's differential equation.

Recall that $\delta=0.05$ so $2 \delta=0.1$.

Now,

$$
\begin{aligned}
& \frac{d}{d t}\left[\begin{array}{l}
2 \\
\sigma_{t}^{(0)} \\
2 \sigma_{t}^{(1)} \\
{ }^{2} \sigma_{t}^{(2)}
\end{array}\right]=\left[\begin{array}{ccc}
\left(2 \delta+\mu_{60+t}^{01}+\mu_{60+t}^{02}\right) & -\mu_{60+t}^{01} & -\mu_{60+t}^{02} \\
-\mu_{60+t}^{10} & \left(2 \delta+\mu_{60+t}^{10}+\mu_{60+t}^{12}\right) & -\mu_{60+t}^{12} \\
0 & 0 & 2 \delta
\end{array}\right]\left[\begin{array}{c}
2 \sigma_{t}^{(0)} \\
2 \sigma_{t}^{(1)} \\
2 \sigma_{t}^{(2)}
\end{array}\right] \\
& -\left[\begin{array}{c}
\sum_{l \neq 0} \mu_{60+t}^{0 l}\left(V_{t}^{(0)}-\left(V_{t}^{(l)}-S_{t}^{(0 l)}\right)\right)^{2} \\
\sum_{l \neq 1} \mu_{60+t}^{1 l}\left(V_{t}^{(1)}-\left(V_{t}^{(l)}-S_{t}^{(1 l)}\right)\right)^{2} \\
\sum_{l \neq 2} \mu_{60+t}^{2 l}\left(V_{t}^{(2)}-\left(V_{t}^{(l)}-S_{t}^{(2 l)}\right)\right)^{2}
\end{array}\right]
\end{aligned}
$$

Also, 


$$
\begin{gathered}
S_{t}^{(01)}=0, S_{t}^{(02)}=5000, S_{t}^{(10)}=0, S_{t}^{(12)}=5000, \\
W_{t}^{j j}=V_{t}^{(j)}-S_{t}^{(j j)}=V_{t}^{(j)}, W_{t}^{00}=V_{t}^{(0)},
\end{gathered}
$$

and

$$
W_{t}^{(j l)}=V_{t}^{(l)}-S_{t}^{(j l)} .
$$

Therefore,

$$
\begin{aligned}
& \frac{d}{d t}\left[\begin{array}{c}
2 \sigma_{t}^{(0)} \\
2 \sigma_{t}^{(1)} \\
2 \sigma_{t}^{(2)}
\end{array}\right]=\left[\begin{array}{ccc}
0.15+0.025 t & -0.05 & -0.025 t \\
-0.025 & 0.125+0.04 t & -0.04 t \\
0 & 0 & 0.1
\end{array}\right]\left[\begin{array}{c}
2 \sigma_{t}^{(0)} \\
2 \sigma_{t}^{(1)} \\
2 \sigma_{t}^{(2)}
\end{array}\right] \\
& -\left[\begin{array}{l}
0.05\left(V_{t}^{(0)}-V_{t}^{(1)}\right)^{2}+0.025 t\left(5000+V_{t}^{(0)}-V_{t}^{(2)}\right)^{2} \\
0.025\left(V_{t}^{(1)}-V_{t}^{(0)}\right)^{2}+0.04 t\left(5000+V_{t}^{(1)}-V_{t}^{(2)}\right)^{2} \\
0
\end{array}\right] .
\end{aligned}
$$

Terminal Conditions are as follows:

$$
{ }^{2} \sigma_{10}^{(0)}={ }^{2} \sigma_{10}^{(1)}={ }^{2} \sigma_{10}^{(2)}=0 .
$$

These two systems of differential equations are coupled and they can be solved together. That is, we can combine the systems to obtain

$$
\begin{aligned}
\frac{d}{d t}\left[\begin{array}{l}
V_{t}^{(0)} \\
V_{t}^{(1)} \\
{ }^{2} \sigma_{t}^{(0)} \\
{ }^{2} \sigma_{t}^{(1)} \\
{ }^{2} \sigma_{t}^{(2)}
\end{array}\right]= & {\left[\begin{array}{ccccc}
0.1+0.025 t & -0.05 & 0 & 0 & 0 \\
-0.025 & 0.075+0.04 t & 0 & 0 & 0 \\
0 & 0 & 0.15+0.025 t & -0.05 & -0.025 t \\
0 & 0 & -0.025 & 0.125+0.04 t & -0.04 t \\
0 & 0 & 0 & 0 & 0.1
\end{array}\right]\left[\begin{array}{c}
V_{t}^{(0)} \\
V_{t}^{(1)} \\
2 \sigma_{t}^{(0)} \\
2 \sigma_{t}^{(1)} \\
2 \sigma_{t}^{(2)}
\end{array}\right] } \\
- & {\left[\begin{array}{l}
-125 t \\
-54.36-200 t) \\
0.05\left(V_{t}^{(0)}-V_{t}^{(1)}\right)^{2}+0.025 t\left(5000+V_{t}^{(0)}-V_{t}^{(2)}\right)^{2} \\
0.025\left(V_{t}^{(1)}-V_{t}^{(0)}\right)^{2}+0.04 t\left(5000+V_{t}^{(1)}-V_{t}^{(2)}\right)^{2} \\
0
\end{array}\right.}
\end{aligned}
$$

Notice that for these models, we have terminal conditions and not initial conditions. The differential equation solvers that are included with Matlab require initial conditions. Thus, we can simply use the substitution $t=10-s$ and the Chain Rule to rewrite the system so that the terminal conditions become initial conditions. Using the Matlab's 'ode45' solver we have the following solutions for this system of differential equations. An identical solution is obtained when the example is presented to Matlab 
in matrix form. Note that one must rewrite the solution back to the variable $t$, once a solution has been obtained. Figure 3 represents the expected values and Figure 4 represents the standard deviations (square root of the variances) for this example.

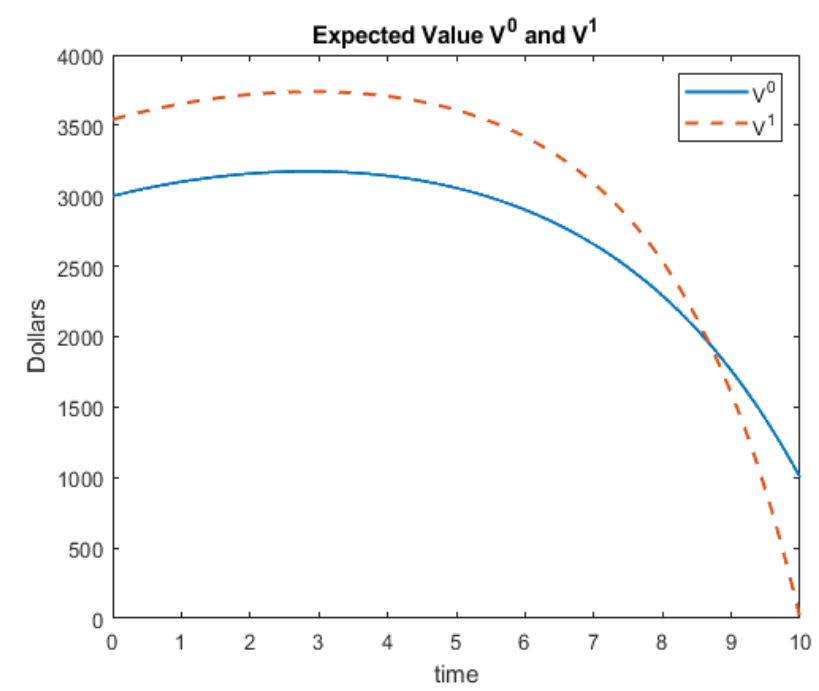

Figure 3

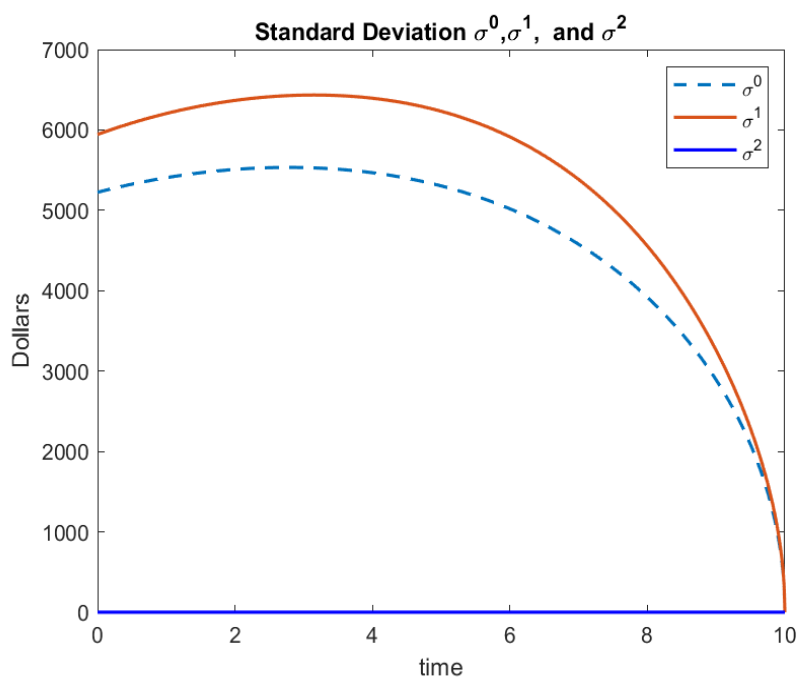

Figure 4

We end the example by stating the following:

(1) We have used Matlab to solve this system of differential equations and acquired both $V_{t} i_{t}$ and ${ }^{2} \sigma_{t}^{(j)}$ in the process.

(2) This step was easily accomplished using MATLAB. 


\subsection{Example 3}

We now consider a six state insurance model. Consider a disability income insurance model with six states: well (State 0), temporary state of disability (State 1), temporary state of disability 2 (State 2), temporary state of disability 3 (State 3), temporary state of disability 4 (State 4), and dead (State 5), as indicated in Figure 5. We also assume that the following transition rates apply to this example:

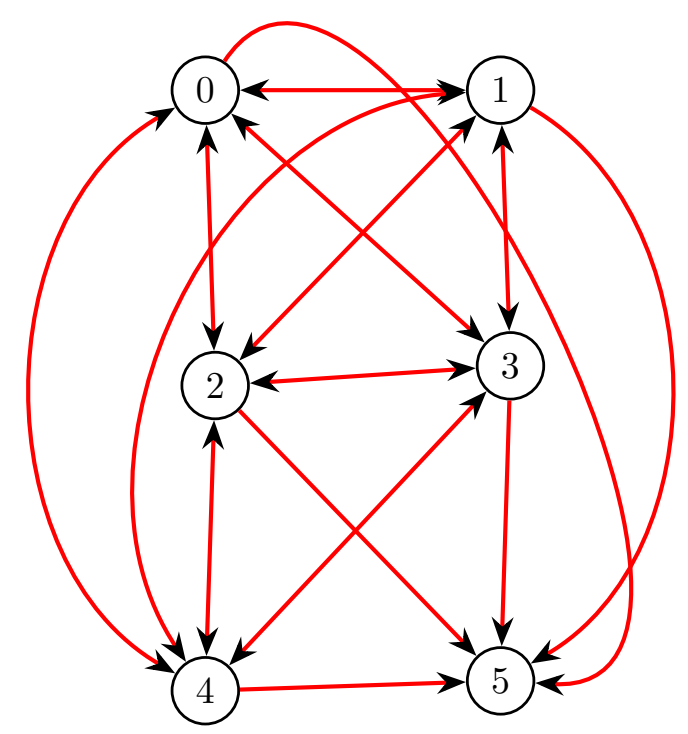

Figure 5

$$
\begin{aligned}
& \mu_{60+t}^{01}=0.01 t, \quad \mu_{60+t}^{02}=0.015, \quad \mu_{60+t}^{03}=0.01, \mu_{60+t}^{04}=0.01 \mu_{60+t}^{05}=0.018 \\
& \mu_{60+t}^{10}=0.06, \quad \mu_{60+t}^{12}=0.01, \quad \mu_{60+t}^{13}=0.020, \quad \mu_{60+t}^{14}=0.01 t \mu_{60+t}^{15}=0.02 t \\
& \mu_{60+t}^{20}=0.055, \quad \mu_{60+t}^{21}=0.15, \quad \mu_{60+t}^{23}=0.025, \quad \mu_{60+t}^{24 t}=0.027, \mu_{60+t}^{15}=0.035, \\
& \mu_{60+t}^{30}=0.05, \quad \mu_{60+t}^{31}=0.045, \quad \mu_{60+t}^{32}=0.015, \quad \mu_{60+t}^{34}=0.018 t, \quad \mu_{60+t}^{35}=0.038, \\
& \mu_{60+t}^{40}=0.04, \quad \mu_{60+t}^{41}=0.045, \quad \mu_{60+t}^{42}=0.025, \quad \mu_{60+t}^{43}=0.028 t, \mu_{60+t}^{45}=0.18 t
\end{aligned}
$$

The valuation force of interest is $5 \%$.

A 10-year health insurance product has the following features: 
- The product is issued to lives aged 60 in the healthy state.

- The product pays a death benefit of $\$ 50,000$ at the moment of death.

- The product pays a continuous disability benefit at a rate of $\$ 2500$ per year while the insured is temporarily disabled.

- Net premiums are payable continuously while the insured is healthy.

- The product pays an endowment of $\$ 5000$ if the person lives beyond 10 years.

You are also given enough information to use the Equivalence Premium Principle to calculate for this group to be offerred at a rate of $P=999.99$.

From this information, we have

$$
\begin{aligned}
& S_{t}^{(01)}=0, S_{t}^{(02)}=0, S_{t}^{(03)}=0, S_{t}^{(04)}=0, S_{t}^{(05)}=50,000 \\
& S_{t}^{(10)}=0, S_{t}^{(12)}=0, S_{t}^{(13)}=0, S_{t}^{(14)}=0, S_{t}^{(15)}=50,000 \\
& S_{t}^{(20)}=0, S_{t}^{(21)}=0, S_{t}^{(23)}=0, S_{t}^{(24)}=0, S_{t}^{(25)}=50,000 \\
& S_{t}^{(30)}=0, S_{t}^{(31)}=0, S_{t}^{(32)}=0, S_{t}^{(34)}=0, S_{t}^{(35)}=50,000 \\
& S_{t}^{(40)}=0, S_{t}^{(41)}=0, S_{t}^{(42)}=0, S_{t}^{(43)}=0, S_{t}^{(45)}=50,000 .
\end{aligned}
$$

By Thiele's differential equation, we have the following:

$$
\begin{aligned}
\frac{d}{d t}\left(V_{t}^{(0)}\right)= & 0.05 V_{t}^{(0)}+999.99-\left\{0.01 t\left(V_{t}^{(1)}-V_{t}^{(0)}\right)+0.015\left(V_{t}^{(2)}-V_{t}^{(0)}\right)\right. \\
& \left.+0.01\left(V_{t}^{(3)}-V_{t}^{(0)}\right)+0.01\left(V_{t}^{(4)}-V_{t}^{(0)}\right)+0.018\left(50,000+V_{t}^{(5)}-V_{t}^{(0)}\right)\right\} \\
= & V_{t}^{(0)}(0.05+0.01 t+0.015+0.01+0.01+0.018)+999.99-0.01 t V_{t}^{(1)}-0.015 V_{t}^{(2)}- \\
& \quad-0.01 V_{t}^{(3)}-0.01 V_{t}^{(4)}-0.018(50,000) \\
= & V_{t}^{(0)}(0.103+0.01 t)-0.01 t V_{t}^{(1)}-0.015 V_{t}^{(2)}-0.01 V_{t}^{(3)}-0.01 V_{t}^{(4)}+99.99
\end{aligned}
$$

Note that $V_{t}^{(5)}=0$ for all $t \geq 0$.

In a similar manner, we have the following:

$$
\frac{d}{d t}\left(V_{t}^{(0)}\right)=-0.06 V_{t}^{(0)}+(0.14+.03 t) V_{t}^{(1)}-0.01 V_{t}^{(2)}-0.02 V_{t}^{(3)}-0.01 t V_{t}^{(4)}+2500+100 t
$$




$$
\begin{gathered}
\frac{d}{d t}\left(V_{t}^{(0)}\right)=-0.055 V_{t}^{(0)}-0.15 V_{t}^{(1)}+0.342 V_{t}^{(2)}-0.25 V_{t}^{(3)}-0.27 V_{t}^{(4)}-4250 \\
\frac{d}{d t}\left(V_{t}^{(0)}\right)=-0.05 V_{t}^{(0)}-0.045 t V_{t}^{(1)}-0.015 V_{t}^{(2)}+(0.198+0.018 t) V_{t}^{(3)}-0.018 t V_{t}^{(4)}-4400 \\
\frac{d}{d t}\left(V_{t}^{(0)}\right)=-0.04 V_{t}^{(0)}-0.045 V_{t}^{(1)}-0.025 V_{t}^{(2)}-0.028 t V_{t}^{(3)}+(0.16+0.208 t) V_{t}^{(4)}-2500+9000 t .
\end{gathered}
$$

In matrix form we have

$$
\begin{aligned}
\frac{d}{d t}\left[\begin{array}{l}
V_{t}^{(0)} \\
V_{t}^{(1)} \\
V_{t}^{(2)} \\
V_{t}^{(3)} \\
V_{t}^{(4)}
\end{array}\right]= & {\left[\begin{array}{ccccc}
(0.103+0.01 t) & -0.01 t & -.015 & -0.01 & -0.01 \\
-.06 & (0.14+.03 t) & -0.01 & -0.02 & -.01 t \\
-0.055 & -0.15 & 0.342 & -0.25 & -0.27 \\
-0.05 & -0.045 t & -0.015 & (0.198+0.018 t) & 0.018 t \\
-0.04 & -0.045 & -0.025 & -0.028 t & (0.16+0.208 t)
\end{array}\right]\left[\begin{array}{c}
V_{t}^{(0)} \\
V_{t}^{(1)} \\
V_{t}^{(2)} \\
V_{t}^{(3)} \\
V_{t}^{(4)}
\end{array}\right] } \\
= & +\left[\begin{array}{l}
99 \\
2500+100 t \\
-4250 \\
-4400 \\
-2500+9000 t
\end{array}\right] \\
= & A\left[\begin{array}{c}
V_{t}^{(0)} \\
V_{t}^{(1)} \\
V_{t}^{(2)} \\
V_{t}^{(3)} \\
V_{t}^{(4)}
\end{array}\right]+B
\end{aligned}
$$

where $A$ and $B$ are used to represent each matrix.

Since $V_{t}^{(5)}=0$ for all $t \geq 0$ we have no need for a differential equation for that particular equation.

$$
\text { Terminal Conditions: }\left\{\begin{array}{l}
V_{10}^{(0)}=5000 \quad \text { (Endowment) } \\
V_{10}^{(1)}=0 \\
V_{10}^{(2)}=0 \\
V_{10}^{(3)}=0 \\
V_{10}^{(4)}=0 \\
V_{10}^{(5)}=0
\end{array}\right.
$$


Now let's consider Hattendorff's differential equation in matrix form.

Recall that $\delta=0.05$ so $2 \delta=0.1$.

Now,

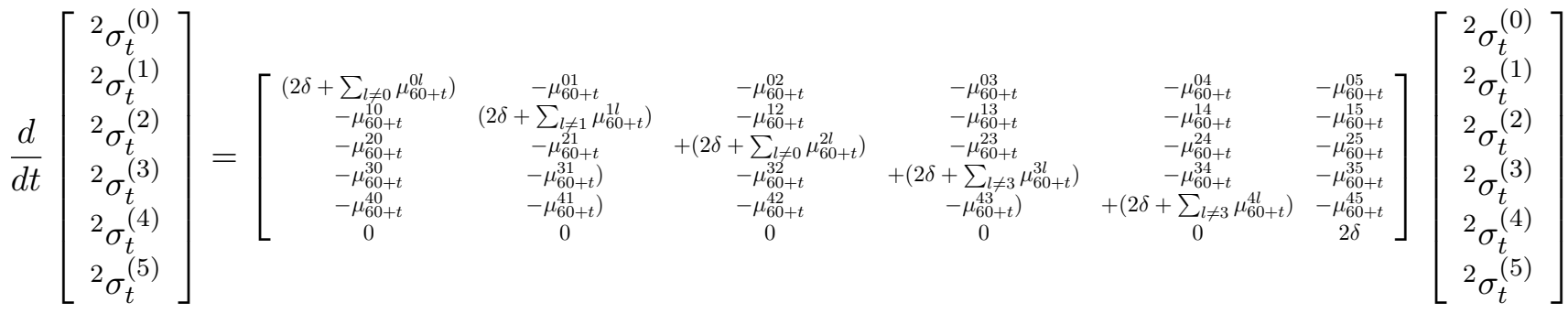

$$
\begin{aligned}
& +\left[\begin{array}{l}
\sum_{l \neq 0} \mu_{60+t}^{0 l}\left(V_{t}^{(0)}-\left(V_{t}^{(l)}-S_{t}^{(0 l)}\right)\right)^{2} \\
\sum_{l \neq 1} \mu_{60+t}^{1 l}\left(V_{t}^{(1)}-\left(V_{t}^{(l)}-S_{t}^{(1 l)}\right)\right)^{2} \\
\sum_{l \neq 2} \mu_{60+t}^{2 l}\left(V_{t}^{(2)}-\left(V_{t}^{(l)}-S_{t}^{(2 l)}\right)\right)^{2} \\
\sum_{l \neq 3} \mu_{60+t}^{0 l}\left(V_{t}^{(0)}-\left(V_{t}^{(l)}-S_{t}^{(0 l)}\right)\right)^{2} \\
\sum_{l \neq 4} \mu_{60+t}^{1 l}\left(V_{t}^{(1)}-\left(V_{t}^{(l)}-S_{t}^{(1 l)}\right)\right)^{2} \\
0
\end{array}\right]
\end{aligned}
$$

Substituting in our known values, we have

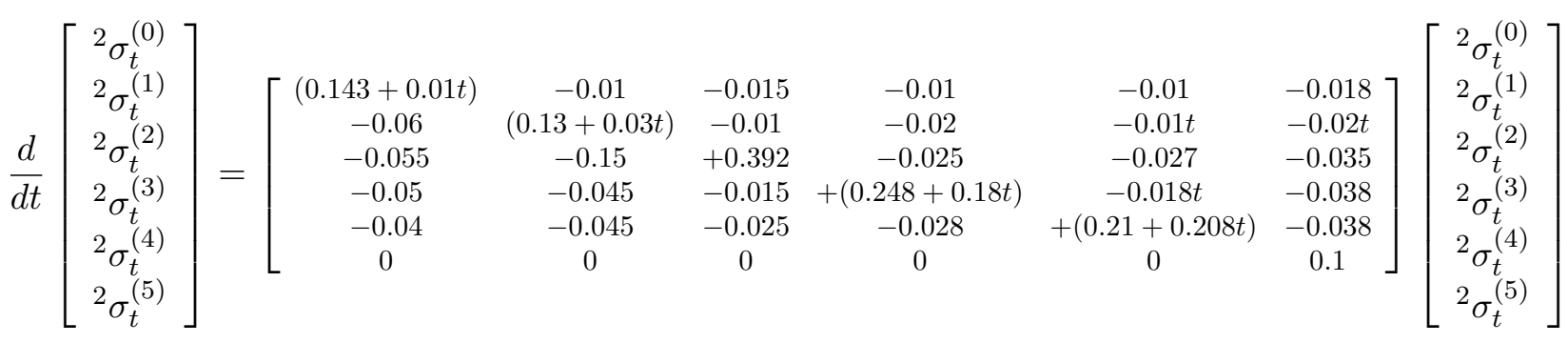

$$
\begin{aligned}
& -\left[\begin{array}{l}
0.01 t\left(V_{t}^{0)}-V_{t}^{(1)}\right)^{2}+0.015\left(V_{t}^{0)}-V_{t}^{(2)}\right)^{2}+0.01\left(V_{t}^{0)}-V_{t}^{(3)}\right)^{2}+0.01\left(V_{t}^{0)}-V_{t}^{(4)}\right)^{2}+0.018\left(50000+V_{t}^{0)}\right)^{2} \\
0.06\left(V_{t}^{1)}-V_{t}^{(0)}\right)^{2}+0.01\left(V_{t}^{1)}-V_{t}^{(2)}\right)^{2}+0.02\left(V_{t}^{1)}-V_{t}^{(3)}\right)^{2}+0.01 t\left(V_{t}^{1)}-V_{t}^{(4)}\right)^{2}+0.02 t\left(50000+V_{t}^{1)}\right)^{2} \\
0.055\left(V_{t}^{2)}-V_{t}^{(0)}\right)^{2}+0.15\left(V_{t}^{2)}-V_{t}^{(1)}\right)^{2}+0.025\left(V_{t}^{2)}-V_{t}^{(3)}\right)^{2}+0.027\left(V_{t}^{3)}-V_{t}^{(4)}\right)^{2}+0.035\left(50000+V_{t}^{2)}\right)^{2} \\
0.05\left(V_{t}^{3)}-V_{t}^{(0)}\right)^{2}+0.045\left(V_{t}^{3)}-V_{t}^{(1)}\right)^{2}+0.015\left(V_{t}^{3)}-V_{t}^{(2)}\right)^{2}+0.018 t\left(V_{t}^{3)}-V_{t}^{(4)}\right)^{2}+0.038\left(50000+V_{t}^{3)}\right)^{2} \\
0.04\left(V_{t}^{4)}-V_{t}^{(0)}\right)^{2}+0.045\left(V_{t}^{4)}-V_{t}^{(1)}\right)^{2}+0.025\left(V_{t}^{4)}-V_{t}^{(2)}\right)^{2}+0.028 t\left(V_{t}^{4)}-V_{t}^{(3)}\right)^{2}+0.018 t\left(50000+V_{t}^{4)}\right)^{2} \\
0
\end{array}\right] \\
& -C\left[\begin{array}{c}
2 \sigma_{t}^{(0)} \\
2 \sigma_{t}^{(1)} \\
{ }^{2} \sigma_{t}^{(2)} \\
2 \sigma_{t}^{(3)} \\
2 \sigma_{t}^{(4)} \\
2 \sigma_{t}^{(5)}
\end{array}\right]+D
\end{aligned}
$$

where $C$ and $D$ are the matrices above. Note that $D$ is in terms of the solution to the first system of equations.

Terminal Conditions are as follows:

$$
{ }^{2} \sigma_{10}^{(0)}={ }^{2} \sigma_{10}^{(1)}={ }^{2} \sigma_{10}^{(2)}={ }^{2} \sigma_{10}^{(3)}={ }^{2} \sigma_{10}^{(4)}={ }^{2} \sigma_{10}^{(5)}=0 .
$$


These two systems of differential equations are coupled and they can be solved togther. That is, we can combine the systems to obtain

$$
\frac{d}{d t}\left[\begin{array}{c}
V_{t}^{(0)} \\
V_{t}^{(1)} \\
V_{t}^{(2)} \\
V_{t}^{(3)} \\
V_{t}^{(4)} \\
2 \sigma_{t}^{(0)} \\
2 \sigma_{t}^{(1)} \\
2 \sigma_{t}^{(2)} \\
2 \sigma_{t}^{(3)} \\
2 \sigma_{t}^{(4)} \\
2 \sigma_{t}^{(5)}
\end{array}\right]=\left[\begin{array}{cc}
A & 0_{5 \times 6} \\
06 \times 5 & C
\end{array}\right]\left[\begin{array}{c}
V_{t}^{(0)} \\
V_{t}^{(1)} \\
V_{t}^{(2)} \\
V_{t}^{(3)} \\
V_{t}^{(4)} \\
2 \sigma_{t}^{(0)} \\
2 \sigma_{t}^{(1)} \\
2 \sigma_{t}^{(2)} \\
2 \sigma_{t}^{(3)} \\
2 \sigma_{t}^{(4)} \\
2 \sigma_{t}^{(5)}
\end{array}\right]+\left[\begin{array}{c}
B \\
D
\end{array}\right]
$$

Notice that for these models, we have terminal conditions and not initial conditions. The differential equations solvers that are included with Matlab are designed to require initial conditions. Thus, we can simply use the substitution $t=10-s$ and the Chain Rule to rewrite the system so that the terminal conditions become initial conditions. Using the Matlab's 'ode45' solver we have the following solutions for this system of differential equations. An identical solution is obtained when the example is presented to Matlab in matrix form. Note that one must rewrite the solution back to the variable $t$, once a solution has been obtained. Figure 6 represents the expected values and Figure 7 represents the standard deviations (square root of the variances) for this example.

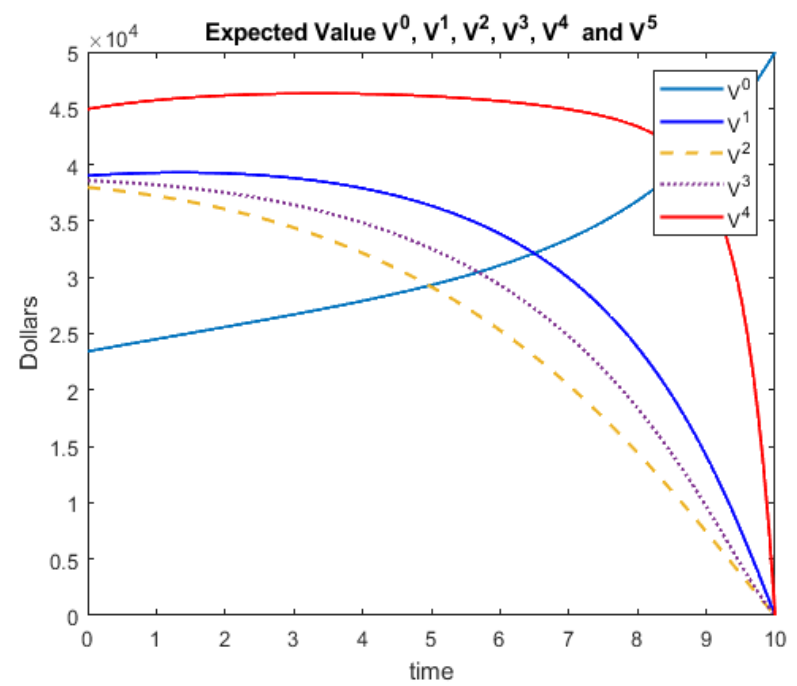

Figure 6 


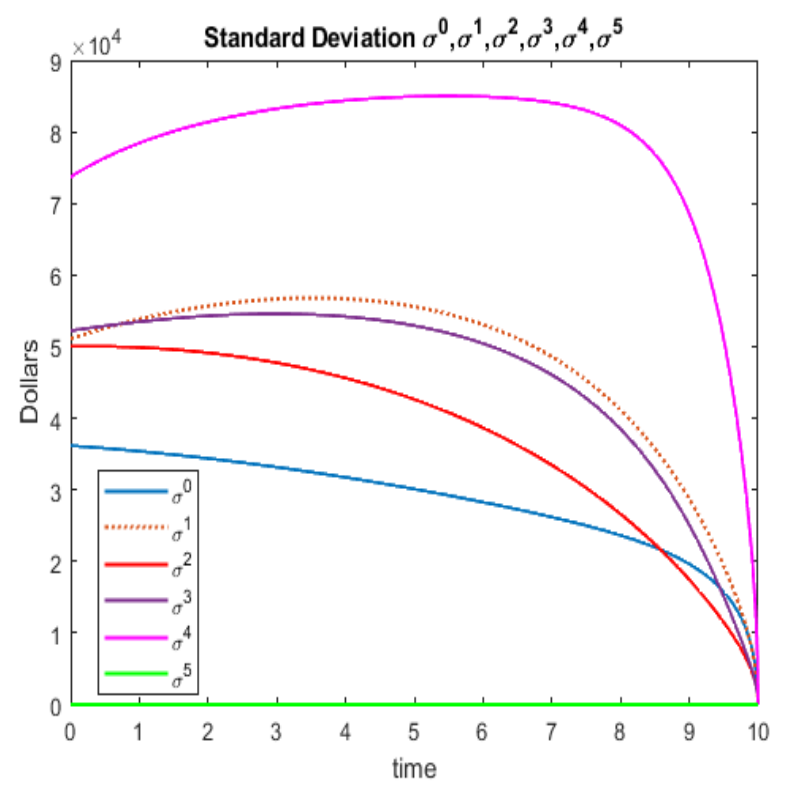

Figure 7

\section{Matlab}

We used Matlab to perform the calculations required to find numerical solutions to three instances of the multi-state model. In each cas, it took Matlab only a fraction of a second to find a solution to our problems.

Matlab can be organized in terms of subroutines or ".m" files. For the examples contained in this paper, we used two files, one to define the transformed system of differential equations and the other, which we called the "driver." The driver was used to call the solver, input the particular system to solve, transform the solution back from variable $s$ to $t$, and then to plot the figures and generate paricular values of system. Matlab has several solvers that are used to solve systems of differential equations. We chose "ode45', a built-in and popular differential equations solver to do the work. These files can be supplied upon request.

\section{Conclusion}

In this paper, we have used the Hattendorff differential equation that we derived in Rajaram and Ritchey (2021). This paper describes the continuous time evolution of the variance of the loss at time $t$ random variable given that it is in state $j$ denoted by ${ }^{2} \sigma_{t}^{(j)}$ for continuous cash flows, along with terminal conditions. In addition, we have also derived a Hattendorff recursion for the annual and $h$-yearly evolution of ${ }^{2} \sigma_{t}^{(j)}$ for annual cash and $h$-yearly cash flows respectively, along with terminal conditions. Both derivations were carried out in the general setting of a multi-state Markov insurance model. In both cases, the equations are coupled with policy values which are in turn solutions of either the Thiele's differential equation in continuous time or a policy value recursion in discrete time. 
We have also demonstrated that solutions for various instances of the problem can be easily approximated using Matlab (or similar software). We have shown the adaptation and closed form expressions for the differential equation and recursion for three examples.

To our knowledge, this is the first time such expressions have been used to solve specific instances for the continuous time and discrete time evolution of ${ }^{2} \sigma_{t}^{(j)}$ for the multi-state case.

\section{References}

Bladt, M., Asmussen, A., \& Steffensen, S. (2020). Matrix representations of life insurance payments. European Actuarial J., 10, 29-67.

Bowers, N. L., Gerber, H. U., Hickman, J. C., Jones, D. A., \& Nesbitt, C. J. (1986). Actuarial mathematics. Illinois: The Society of Actuaries.

Dickson, C. M. D., Hardy, M. R., \& Waters, H. R. (2020). Actuarial mathematics for life contingent risks. Cambridge University Press.

Gerber, H. U. (1979). An introduction to mathematical risk theory. Univ. of Pennsylvania, Philadelphia: Huebner Foundation.

Gerber, H. U. (1986). Lebellsversicherungsmathematik. Berlin, Heidelberg, New York: Springer-Verlag.

Hattendorff, K. (1868). Das risiko bei der lebenversicherung. Masius Rundschau der Versi-, 18, 169-183.

Norberg, R. (1992). Hattendorff's theorem and thiele's differential equation generalized. Scand. Actuarial J., 1992, 1:2-14.

Papatriandafylou, A., \& Waters, H. R. (1984). Martingales in life insurance. Scand. Actuarial J., $1984,210-230$.

Rajaram, R., \& Ritchey, N. (2021). Hattendorff differential equation for multi-state markov insurance models. Risks, 9, 169-187.

Ramlau-Hansen, H. (1988). Hattendorff's theorem: a markov chain and counting process approach. Scand. Actuarial J., 1987, 143-156. 